\title{
Automatic and Rapid Calibration of Urban Building Energy Models
}

\author{
Yixing Chen ${ }^{1,2}$, Tianzhen Hong ${ }^{2}$ \\ ${ }^{1}$ College of Civil Engineering, Hunan University, Changsha 410082, China \\ ${ }^{2}$ Building Technology and Urban Systems Division, Lawrence Berkeley National Laboratory, \\ Berkeley, CA 94720, USA
}

\begin{abstract}
Urban building energy modeling (UBEM) is attracting increasing attention in the energy modeling filed. Unlike modeling a single building using detailed building systems information, UBEM generally uses existing limited building stock data consisting of high-level building information. Default assumptions are made for the building systems based on that information, such as building type, size, and vintage. This study introduced a novel method of automatic and rapid calibration of UBEM based on the annual site and source energy use by learning the correlations between key model input parameters and the building energy use from USDOE reference buildings. A case study was presented to calibrate 112 large office buildings built before 1978 in San Francisco. An EnergyPlus model was created for each building. Fifteen model parameters were selected to calibrate the models. The Monte Carlo sampling was used to create 1000 samples to represent the parameter combination space reasonably. Then 1000 simulations were performed for the DOE reference building for large office built before 1978 in San Francisco to create an energy performance database. The results showed that by learning from the energy performance database, it took 2.7 simulation runs on average to calibrate each building model rapidly. After the calibration, the distribution of each parameter was obtained to replace the single default value. For example, the lighting power densities after calibration were $9.87 \mathrm{~W} / \mathrm{m}^{2}$ on average, very different from the default value of $21.39 \mathrm{~W} / \mathrm{m}^{2}$. The case study successfully demonstrated the effectiveness of the novel calibration method for UBEM in the mild climate. For future work, the method needs to be tested in other climate zones and for other building types.
\end{abstract}

\section{Introduction}

Today's cities consume more than two-thirds of the world's primary energy and account for more than $70 \%$ of global greenhouse gas (GHG) emissions. The building sector in cities can consume up to $75 \%$ of the total primary energy. In the United States, half of the commercial buildings and $55 \%$ of the residential buildings were built before 1980 , while $82 \%$ of both commercial and residential buildings were built before 2000 (U.S. Environmental Protection Agency, 2018a, 2018b). Retrofitting the existing building stock to improve energy efficiency and reduce energy use is a crucial strategy for cities to reduce GHG emissions and mitigate climate change.

Urban Building Energy Modeling (UBEM) refers to the application of bottom-up physics-based building energy models to predict operational energy use as well as indoor and outdoor environmental conditions for groups of buildings in an urban context (Reinhart and Davila, 2016). UBEM is an excellent tool to explore opportunities for energy conservation measures (ECMs) when applying to a large group of buildings in the urban context (Chen et al., 2017). UBEM can also be used to evaluate the district-scale technologies (Yamaguchi et al., 2007). Unlike modeling a single building, which will use detailed information, UBEM generally uses existing building stock data consisting of high-level building information (Chen et al., 2019). The details of building systems are rarely available for a large number of buildings at the city scale. Typically, the building systems and their efficiency values are determined based on the building type, vintage, and size, which represent the average conditions among peer groups (Hong et al., 2020).

The use of archetype may lead to smaller ranges of site and source energy use intensities (EUIs) distribution for simulated results compared with measured data (Chen and Hong, 2018). Many cities in the United States begin to enforce energy ordinance for existing buildings. Those energy data can be used to calibrate the UBEM and reduce the difference between simulated results and measured data. The model calibration is commonly defined as an inverse approximation because of the need for tuning necessary inputs to reconcile the outputs by a simulation program, as closely as possible to the measured energy data (Yang and Becerik-Gerber, 2015). There are several automated calibration methods developed to calibrate individual building, including optimization-based methods (O'Neill and Eisenhower, 2013), pattern or rule-based methods (Sun et al., 2016), and Bayesian calibration methods (Lim and Zhai, 2017). Those technologies are well demonstrated for individual buildings. Although they can be directly applied to calibrate UBEM, the number of simulations required to calibrate the UBEM is proportional to the number of buildings, which is a huge number of simulations.

When simplified models (e.g., resistance-capacitance network models) was used in UBEM, each simulation run may only take several milliseconds or less. So 
complex calibration methods such as Bayesian calibration can be (1) Calibration parameters selection
and Monte Carlo sampling

(2) Energy performance database of reference building

(3) Guess candidates

(4) Determine validated solutions

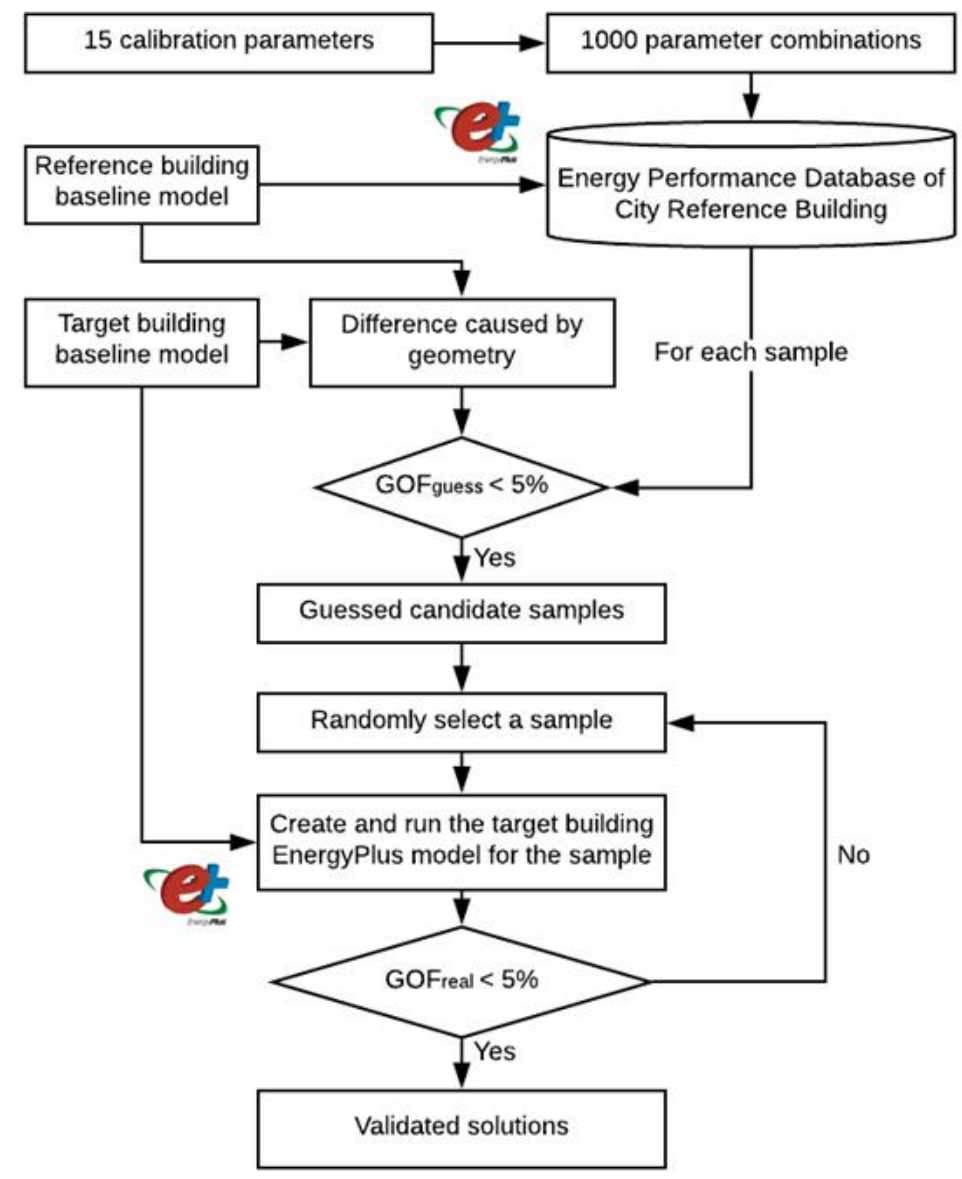

Figure 1: Workflow of the UBEM calibration.

Table 1: Selected 15 key parameters and their value options.

\begin{tabular}{|c|c|c|c|c|c|}
\hline ID & Name (Unit) & Option 1 & Option 2 & Option 3 & Option 4 \\
\hline OCD & Occupancy density (person $/ \mathrm{m}^{2}$ ) & 0.05 & 0.13 & 0.22 & 0.3 \\
\hline LPD & Lighting power density $\left(\mathrm{W} / \mathrm{m}^{2}\right)$ & 6.46 & 13.58 & 20.69 & 27.81 \\
\hline EPD & Equipment power density $\left(\mathrm{W} / \mathrm{m}^{2}\right)$ & 8.91 & 12.3 & 15.69 & 19.08 \\
\hline OAF & Outdoor air flow rate (L/s ·person) & 5.56 & 8.33 & 11.11 & 13.89 \\
\hline INF & Infiltration rate $\left(\mathrm{L} / \mathrm{s} \cdot \mathrm{m}^{2}\right.$ wall area $)$ & 0.30 & 0.58 & 0.86 & 1.13 \\
\hline WWR & Windows to wall ratio & 0.25 & 0.38 & 0.52 & 0.65 \\
\hline FAN & Fan efficiency & 0.65 & 0.61 & 0.57 & 0.54 \\
\hline COP & Cooling COP & 5.15 & 4.46 & 3.76 & 3.07 \\
\hline $\mathrm{EFF}$ & Heating efficiency & 0.95 & 0.89 & 0.84 & 0.78 \\
\hline SET & Temperature setpoint $\left({ }^{\circ} \mathrm{C}\right)$ & $19-26$ & $20-25$ & $21-24$ & \\
\hline $\mathrm{SCH}$ & Operational schedule (days) & 4 & 5 & 6 & $6+$ \\
\hline SHW & Water heater type and efficiency & Gas, 0.93 & Gas, 0.85 & Gas, 0.78 & $\mathrm{HP}, 3.3$ \\
\hline $\mathrm{ECO}$ & Air economizer & Yes & No & & \\
\hline DLT & Daylighting control & Yes & No & & \\
\hline WIN & $\begin{array}{l}\text { Window glazing [SHGC, U-Factor } \\
\left(\mathrm{W} / \mathrm{m} \cdot{ }^{\circ} \mathrm{C}\right) \text {, Visible transmittance] }\end{array}$ & $\begin{array}{r}{[0.18,1.43} \\
0.42]\end{array}$ & $\begin{array}{r}{[0.46,2.67} \\
0.81]\end{array}$ & $\begin{array}{r}{[0.61,4.37} \\
0.81]\end{array}$ & $\begin{array}{r}0.82,6.98 \\
0.81]\end{array}$ \\
\hline
\end{tabular}

COP: coefficient of performance, SHGC: solar heat gain coefficient, U-Factor: overall heat transfer coefficient, 
6+: 6 working days and HVAC always on, HP: heat pump water heater

applied (Kristensen et al., 2018). However, for detailed physics-based energy models, it may take several minutes or more to run a simulation. It is therefore very important to reduce the number of simulations. Nagpal et al. (Nagpal et al., 2019) showed a methodology for autocalibrating UBEM using surrogate modeling techniques. 200 training samples are sufficient to develop robust statistical surrogate models with an optimization algorithm to estimate the properties of unknown building parameters.

However, the buildings in the UBEM are similar. The UBEM calibration should not be the simple scale-up of the methods used for individual buildings. Another challenge for calibrating the UBEM is the limited availability of energy use data: usually, only annual energy consumption data (site and/or source) is available for buildings at a large scale. This is in contrast to calibrating individual building energy models, which usually have monthly electricity and gas consumption data.

This study develops a novel method by learning from city reference buildings to calibrate 112 large office buildings built before 1978 in San Francisco automatically and rapidly based on the reported annual site and source EUIs.

\section{Method}

The proposed UBEM calibration method includes four major steps (Figure 1). The first step is to identify a list of unknown parameters, which significantly influence building energy performance. In this study, fifteen parameters are selected with two to four value options as shown in Table 1. The full combinations of the parameters lead to a massive number of simulations. The Monte Carlo Sampling technology is used to create 1000 samples as a reasonable representation of the parameter combinations.

The second step is to create the energy performance database of the DOE reference building. One EnergyPlus model of the reference building is created for each parameter combination sample. The 1000 simulation results of the reference building are stored to create the energy performance database.

The third step is to guess the candidate samples without running EnergyPlus simulations. For a target building, the baseline results (building geometry with the archetype parameters) are simulated. The baseline results of the target building and the reference building are used to calculate the difference caused by the geometry in Equations (2) and (3). The guessed good of fitness (GOF) is calculated based on Equation (1). In this study, the GOF threshold is set to $5 \%$.

$G O F_{\text {guess }}=\frac{\sqrt{\frac{w_{e}^{2} \times\left(y_{e}-\alpha_{e} \times \hat{p}_{e}\right)^{2}+w_{g}^{2} \times\left(y_{g}-\alpha g \times \hat{p}_{g}\right)^{2}}{w_{e}^{2}+w_{g}^{2}}}}{\left(w_{e} \times y_{e}+w_{g} \times y_{g}\right)}$

$\alpha_{e}=\frac{\text { Target building baseline electricity use intensity }}{\text { Prototype baseline electricity use intensity }}$ $\alpha_{g}=\frac{\text { Target building baseline gas use intensity }}{\text { Prototype baseline gasuse intensity }}$

where $w_{e}$ is the weight factor for electricity (3.14, source factor), $w_{g}$ is the weight factor for natural gas (1.05, source factor), $y_{e}$ is the measured electricity use intensity, $y_{g}$ is the measured natural gas use intensity, $\hat{p}_{e}$ is the simulated prototype electricity use intensity, $\hat{p}_{g}$ is the simulated prototype natural gas use intensity, $\alpha_{e}$ is the electricity use intensity adjustment factor, $\alpha_{g}$ is the natural gas use intensity adjustment factor.

Moreover, the last step is to determine the valid solution(s). A sample from the guessed candidate samples is randomly selected and one EnergyPlus model of the target building is created based on the sample parameter values. The real GOF is calculated based on Equation (4). When the real GOF is less than 5\%, the solution is deemed adequate and valid.

$G O F_{\text {real }}=\frac{\sqrt{\frac{w_{e}^{2} \times\left(y_{e}-\hat{y}_{e}\right)^{2}+w_{g}^{2} \times\left(y_{g}-\hat{y}_{g}\right)^{2}}{w_{e}^{2}+w_{g}^{2}}}}{\left(w_{e} \times y_{e}+w_{g} \times y_{g}\right)}$

where $\hat{y}_{e}$ is the simulated electricity use intensity of the target building for the sample, and $\hat{y}_{g}$ is the simulated natural gas use intensity of the target building for the sample.

To test the performance of the proposed method, all the samples in the guessed solutions are tested. The average number (expected value) of simulation runs to calibrate a building is calculated using Equation (5).

ClibrationRuns $_{t b}=\frac{\text { Number of Guessed Solutions }}{\text { Number of validated Solutions }}$

\section{Result}

For buildings subject to the city's energy benchmarking and disclosure ordinance, either annual electricity use intensity and annual natural gas use intensity are available, or the annual site and source EUIs are available, which can be used to back-calculate the annual electricity and natural gas use by assuming the source factors using Equations (6) and (7), or vice versa. Figure 2 shows the site and source EUIs of the reference building for all the 1000 samples sorted by the site EUI. Figure 3 shows the measured site and source EUIs of the target buildings for calibration sorted by site EUI. The samples/buildings in Figures 2 and 3 are sorted based on the site EUI, so there are some oscillations in the source EUI results.

$y_{\text {source }}=w_{e} \times y_{e}+w_{g} \times y_{g}$

$y_{\text {site }}=y_{e}+y_{g}$

Where $y_{\text {source }}$ is the source EUI and $y_{\text {site }}$ is the site EUI. 

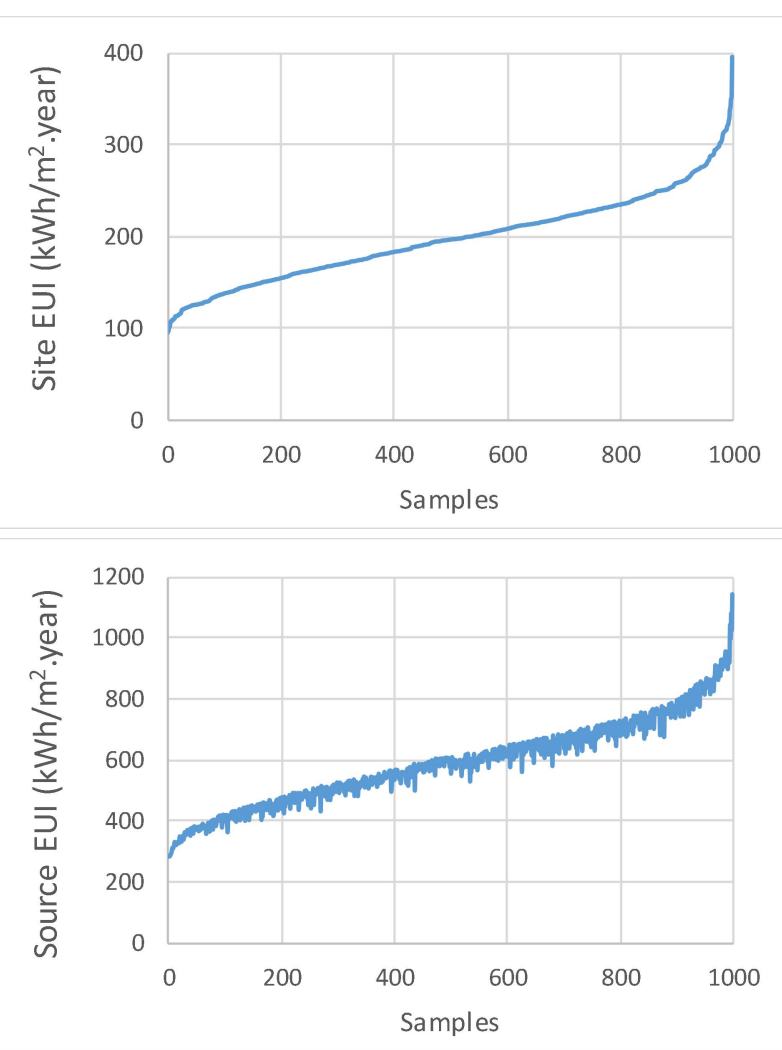

Figure 2: Distributions of the site and source EUIs of the reference building results.

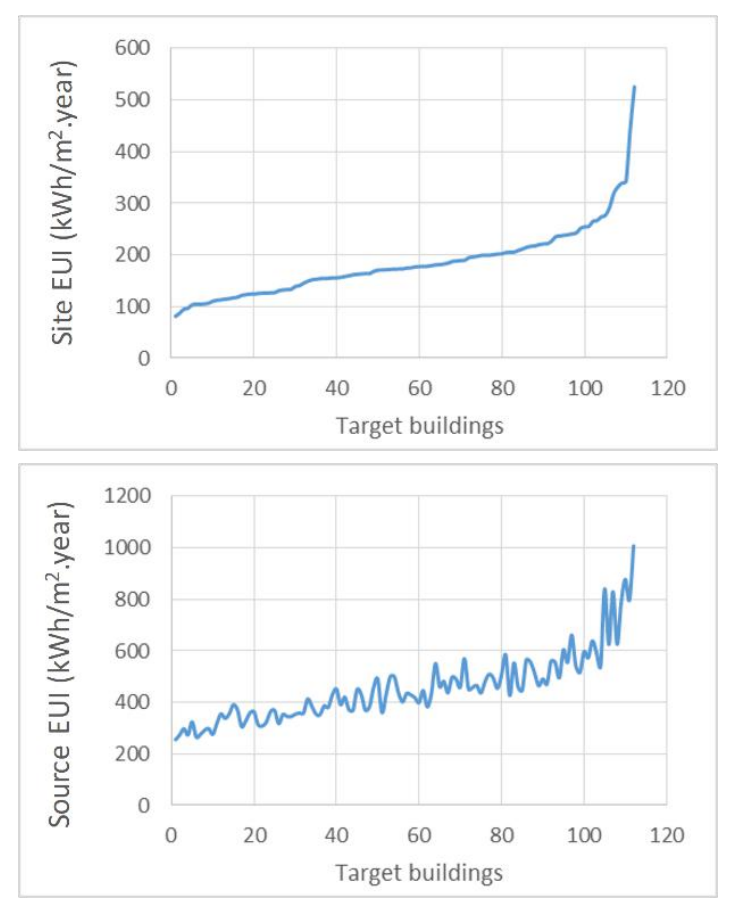

Figure 3: Measured site and source EUIs of the target buildings for calibration.

Figure 4 shows the distribution of the number of guessed solutions. About half of the buildings have more than 100 guessed solutions. Figure 5 shows the average number of EnergyPlus runs to calibrate each building. 97 buildings $(87 \%)$ only need to run one or two simulations to calibrate the model. The expected value of the total simulations to calibrate the 112 buildings is 191.4, equivalent to 1.7 simulation runs per building on average. In addition, each building also needs to run a baseline simulation, which makes the total 2.7 simulation runs to calibrate each building on average.

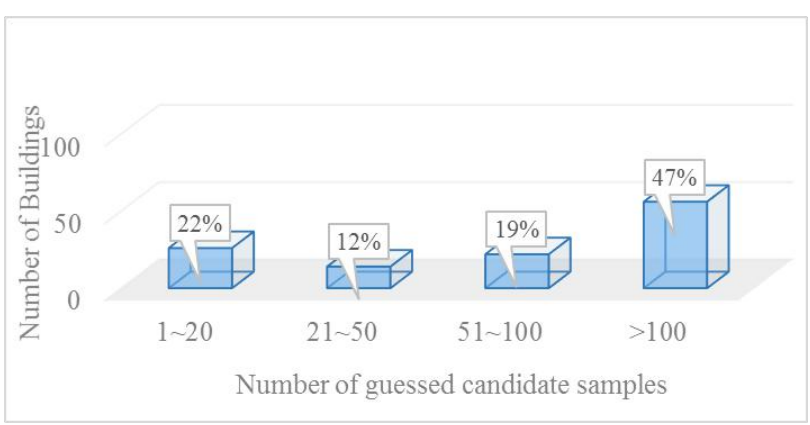

Figure 4. Distribution of the number of guessed candidate samples

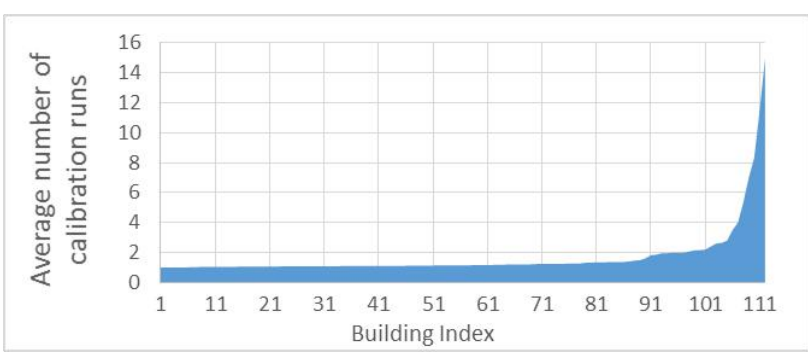

Figure 5. Average number of calibration runs per building

The 112 buildings belong to the same archetype based on their building type, size, and vintage. In previous studies (Chen et al., 2017; Chen and Hong, 2018), these buildings are assigned with the same values for the selected parameters. Table 2 shows the distributions of the parameter values after calibration as well as the default and the calibrated average values. The calibrated values of lighting power density and equipment power density are much smaller than the default archetype values with more than $60 \%$ of the valid solutions using option 1 value. This will strongly impact the retrofit saving estimation of energy conservation measures (ECMs) for lighting and plug load systems. About half of the buildings have air economizers and daylighting control sensors.

\section{Discussion}

As shown in Figure 4, most of the buildings have multiple candidate models. Some of them $(47 \%)$ even have more than 100 candidate models. For each calibration runs, different candidate models may be selected, which may result in different parameter distributions after calibration. The problem will be worse when calibrating only a few buildings. In the future, we will perform multiple calibration runs to check whether the parameter distribution can converge to a unique set. 
Table 2: Parameters values after calibration

\begin{tabular}{|c|c|c|c|c|c|c|c|}
\hline ID & Option 1 & Option 2 & Option 3 & Option 4 & $\begin{array}{l}\text { Default } \\
\text { value }\end{array}$ & $\begin{array}{l}\text { Calibrated } \\
\text { average }\end{array}$ & $\begin{array}{l}\text { Percentage } \\
\text { difference }\end{array}$ \\
\hline OCD & $27 \%$ & $36 \%$ & $21 \%$ & $16 \%$ & 0.11 & 0.15 & $44 \%$ \\
\hline LPD & $66 \%$ & $23 \%$ & $7 \%$ & $3 \%$ & 21.39 & 9.87 & $-54 \%$ \\
\hline EPD & $61 \%$ & $24 \%$ & $11 \%$ & $5 \%$ & 14.68 & 10.91 & $-26 \%$ \\
\hline OAF & $21 \%$ & $29 \%$ & $31 \%$ & $20 \%$ & 7.08 & 9.69 & $37 \%$ \\
\hline INF & $26 \%$ & $24 \%$ & $25 \%$ & $25 \%$ & 1.13 & 0.72 & $-37 \%$ \\
\hline WWR & $27 \%$ & $27 \%$ & $23 \%$ & $24 \%$ & 0.38 & 0.44 & $16 \%$ \\
\hline FAN & $24 \%$ & $21 \%$ & $27 \%$ & $28 \%$ & 0.60 & 0.59 & $-2 \%$ \\
\hline COP & $28 \%$ & $22 \%$ & $27 \%$ & $23 \%$ & 5.11 & 4.15 & $-19 \%$ \\
\hline EFF & $23 \%$ & $24 \%$ & $25 \%$ & $28 \%$ & 0.76 & 0.86 & $13 \%$ \\
\hline SET & $31 \%$ & $30 \%$ & $39 \%$ & & Option 3 & \multirow{6}{*}{$\begin{array}{c}\text { Multiple } \\
\text { option values, } \\
\text { average is not } \\
\text { applicable }\end{array}$} & \\
\hline $\mathrm{SCH}$ & $41 \%$ & $31 \%$ & $16 \%$ & $12 \%$ & Option 2 & & \\
\hline SHW & $29 \%$ & $24 \%$ & $23 \%$ & $24 \%$ & Option 3 & & \\
\hline ECO & $54 \%$ & $46 \%$ & & & Option 2 & & \\
\hline DLT & $56 \%$ & $44 \%$ & & & Option 2 & & \\
\hline WIN & $28 \%$ & $25 \%$ & $26 \%$ & $21 \%$ & Option 4 & & \\
\hline
\end{tabular}

\section{Conclusion}

The case study successfully demonstrated the effectiveness of the novel calibration method for a group of large office buildings in the mild climate. By learning from the energy performance database of city reference buildings, it took about 2.7 simulation runs on average to calibrate each building model. After the calibration, the distribution of each parameter was obtained to replace the single default value derived from building vintage and corresponding energy code. For future work, the method needs to be tested in other climate zones and for other building types. We will also apply the calibrated models to evaluate the energy saving potential of ECMs compared with the default values.

\section{Acknowledgment}

The work is funded by Lawrence Berkeley National Laboratory through the Laboratory Directed Research and Development Program and the Assistant Secretary for Energy Efficiency and Renewable Energy, the U.S. Department of Energy under Contract No. DE-AC0205CH11231. The work is also supported by the Scientific Research Foundation provided by Hunan University in China.

\section{References}

Chen, Y., Hong, T., 2018. Impacts of building geometry modeling methods on the simulation results of urban building energy models. Appl. Energy 215, 717-735.

https://doi.org/10.1016/j.apenergy.2018.02.073
Chen, Y., Hong, T., Luo, X., Hooper, B., 2019.

Development of city buildings dataset for urban building energy modeling. Energy Build. 183, 252-265.

https://doi.org/10.1016/j.enbuild.2018.11.008

Chen, Y., Hong, T., Piette, M.A., 2017. Automatic generation and simulation of urban building energy models based on city datasets for city-scale building retrofit analysis. Appl. Energy 205, 323335.

https://doi.org/10.1016/j.apenergy.2017.07.128

Hong, T., Chen, Y., Luo, X., Luo, N., Lee, S.H., 2020.

Ten questions on urban building energy modeling. Build. Environ. 168, 106508.

https://doi.org/10.1016/j.buildenv.2019.106508

Kristensen, M.H., Hedegaard, R.E., Petersen, S., 2018. Hierarchical calibration of archetypes for urban building energy modeling. Energy Build. 175, 219-234.

https://doi.org/10.1016/j.enbuild.2018.07.030

Lim, H., Zhai, Z.J., 2017. Comprehensive evaluation of the influence of meta-models on Bayesian calibration. Energy Build. 155, 66-75. https://doi.org/10.1016/j.enbuild.2017.09.009

Nagpal, S., Mueller, C., Aijazi, A., Reinhart, C.F., 2019. A methodology for auto-calibrating urban building energy models using surrogate modeling techniques. J. Build. Perform. Simul. 12, 1-16. https://doi.org/10.1080/19401493.2018.1457722

O’Neill, Z., Eisenhower, B., 2013. Leveraging the analysis of parametric uncertainty for building 
energy model calibration. Build. Simul. 6, 365377. https://doi.org/10.1007/s12273-013-0125-8

Reinhart, C.F., Davila, C.C., 2016. Urban building energy modeling - A review of a nascent field. Build. Environ. 97, 196-202.

Sun, K., Hong, T., Taylor-Lange, S.C., Piette, M.A., 2016. A pattern-based automated approach to building energy model calibration. Appl. Energy 165, 214-224.

https://doi.org/10.1016/j.apenergy.2015.12.026

U.S. Environmental Protection Agency, 2018a. Commercial Buildings Energy Consumption Survey (CBECS) [WWW Document]. URL https://www.eia.gov/consumption/commercial (accessed 3.10.18).
U.S. Environmental Protection Agency, $2018 b$. Residential Energy Consumption Survey (RECS) [WWW Document]. URL

https://www.eia.gov/consumption/residential/ (accessed 3.10.18).

Yamaguchi, Y., Shimoda, Y., Mizuno, M., 2007. Transition to a sustainable urban energy system from a long-term perspective: Case study in a Japanese business district. Energy Build. 39, 1-12. https://doi.org/10.1016/j.enbuild.2006.03.031

Yang, Z., Becerik-Gerber, B., 2015. A model calibration framework for simultaneous multi-level building energy simulation. Appl. Energy 149, 415-431. https://doi.org/10.1016/j.apenergy.2015.03.048 